La cale sèche mesure 14 mètres de longucur et $45^{\mathrm{m}} 7^{5}$ de large. Le remplissage et la vidange ont lieu sans pompes ; lc terrain situć entre la cale sèche ct la rive c:t occupé par les alcliers de réparation.

Usine moro-ślectripue. - L'usine généraluice est construite à l'extrémité ouest du barrage parallèlement à la rive, sur une longueur de 524 mètres et une largeur totale de 40 mòtres.

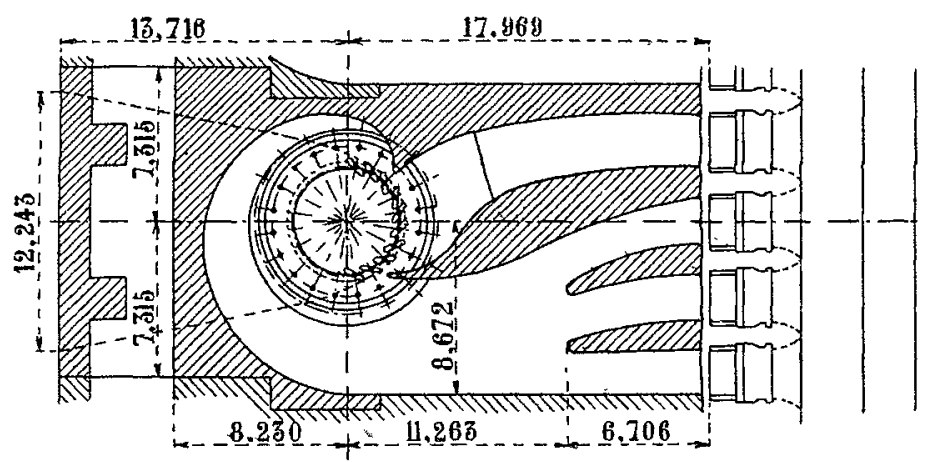

FIG. 2. - COUPE hORIZONTALE DE L'INFRASTRUCTURE PAR UNE TURBINE.

L'infrastructure, dans laquelle sont logées les turbines, a une hautcur de 2 I mètres, depuis la base de la fondation. La superstructure, d'une hauteur de 32 mètres, comprend deux ćlages. Le premier est divisé en deux parties dans le sens de la longueur. D'un côté, la salle des alternateurs ; de l'autre, la salle des transformateurs. La salle' des alternateurs' est desservie par un pont roulant de 150 tonnes. Au deuxième étage sont installés les interrupteurs et l'appareillage éloctrique.

L'infrastructure comprend la chambre d'alimentation qui occupe toute la longueur de l'usine et qui contient les grilles de nettoyage. L'admission de l'eau est faile dans chaque turbine, par quatre vannes alimentées par quatre canaux (fig. 2). Au débouché, ces canaux ont une section de $6^{m} 60 \times 2^{m} 25$. En outre, deux canaux de purge permettent d'évacuer les corps flottants et les glaces.

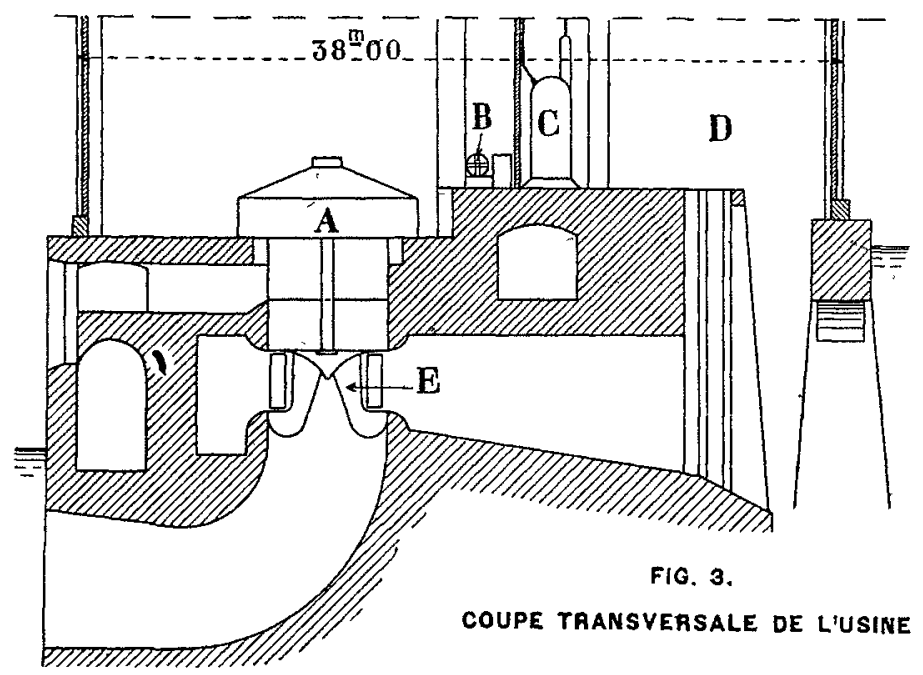

Turbines. - Les turbines de l'usine (fig. 3) ont été établies pour une puissance unitaire de ro.ooo chevaux, au nombre de $3 o$, fce qui donne les 300.000 chevaux sous la chute normale. Elles ne comportent qu'une seule couronne mobile, dont le diamètre est de $4^{\mathrm{m}} 75$. L'eau utilisée est évacuée par un tube d'aspiration de $5^{\mathrm{m}} 5 \mathrm{o}$ de diamètre. La couronne mobile comporte 20 aubages et pèse 65 tonnes. Les aubages distributeurs articulés sur des axes creux el commandés par le régulateur, sont en acier coulé. L'arbre principal à un diamètre de 635 millimètres, est creux avec vide de 200 mil- limètres. Le poids total de $230^{\prime}$ tonnes environ des parties tournantes est équilibré par un pivot combiné pour fonctionner soit à hulle sous pression, soit par roulcaux. La pression de l'huile est de 16 kilos par centimètre carré el lorsqu'elle dimimue, la charge est supportée par les rouleaux. I,s rotors ont été, suivant les constructeurs, fondus les uns en unc seule pièce, les autres en quatre parties. Dans ce dernier cas, le poids du rotor a été augmenté de 25 tonnes par les assemblages solidarisant les quatre pièces. Le poids total d'une turbine est de 450 à 475 tonnes.

La vitesse du rotor est de 57 tours par minule. L'arrivée d'cau à la turbine se fait par quatre ouvertures munies de vannes. L'une d'elles (fig'. 2) alimente un canal n'utilisant que le quart du débit, n'agissant que sur un quart de la circonférence, afin d'éviter les tourbillons dont la formation a été reconnue, et dont l'action se traduisait par l'éloignement de l'axe de la turbine d'un quart du débit; il en résultait un déséquilibre qui ést diminué par co dispositif.

Des essais effecturs sur des modèles de ces turbines ont donné un rendement de 88 pour 100.

La puissance dies machines peul varier de 6.000 chevaux sous une chutc de 6 mètres, à 14.000 chevaux sous uné chute de $1 \mathrm{I}^{\mathrm{m}} \mathrm{po}$.

Les constructeurs sont la Morris $\mathrm{C}^{\circ}$, de Pbiladelphie t la Weltman Senver Morcan, de Cleveland, pour les turbinas el la Lombard Governor $\mathrm{C}^{\circ}$, pour les régulateurs.

Le courant est produit par des alternateurs triphasćs I i.0oo volts 25 périodes, type ì axe vertical ; la tension est élevée à I ro.000 volts dans lo poste transformateur et transportée à cétte tension.

Pierre GuIEu, Ingénieur E.P.C.

\section{L'AMÉNAGEMENT DES MONTAGNES}

\section{I. - L'INFLUENCE DU REBOISEMENT SUR L'ABONDANCE DES EAUX (')}

Sous ce tilre, qui ressemble à celui d'une fable, nòus groupons un travail fortement documenté, divisé en trois chapilres, dont chacun d'eux est la reproduction d'études de M. Paul Descombes, le très courageux insligateur de l'œuvre si féconde que réalise L'Association centrale pour l'Aménagement des Montagnes.

- L'utilisation des eaux est devenue le problème capital de la science française depuis qu'elle a trouvé dáns le transport électrique de leur énergie le moyen de suppléer à l'insuffisance de nos gisements oarbonilères. Et la crise du charbon produite par les hostilités fait voir aux plus aveugles combien il est regrettable que l'irrégularité des eaux débitées par la plupart des bassins n'ait pas permis d'aménager 'un plus grand nombre de chules pour la production des forces motrices.

Sachant que l'Arbre est 10 grand Régulateur des eaux, Ia houille blanche unit ses cfforts à ceux de la navigation et des régions menacées par l'inondation pour réclamer le reboisement des montagnes, auquel l'Administration des Eaux et Forêts consacre sa science et son inlassable dévoutement. Le reboiscment n'a pas d'ailleurs pour seul effel de régulariscr lc débit des eaux, mais il contribuc à leur donnè

(1) Extrait de La Revue Scienlifique du 24 août 1918. 
la clarlé indispensable pour leur cmploi dans les turbines, ct agit sur leur abondance en diminuant leur absorption, cn augmentant aussi la quantité déposée sùr le sol par les phénomènes météorologiques.

Absorprion. - La réduclion que le reboiscment apporte aux déperditions soulerraines a spécialement été sıgnalée par les spéléologistes : "L'un des principaux résultats de ma huitième campagne souterraine effocluée on Irlande el en Angleterre, dit M. Martel, a été de faire constater qu'un reboisement intense, en reconstituant peu à peu la terre végétale, arriverait avec l'aide du temps à oblitérer toutes les fissures d'absorption exiguës des terrains calcaires; les formations de ce genre, si abondantes dans le Midi et l'Est de la France (Causses, Provence, Jura, etc., etc.), sont craquelées au point que les eáux des pluies y passent comme i travers un crible et ne laissent qúe des.déserts de picrres sur une partie de notre terriloirc (1)."

Puures. - L'influence des forêts sur l'abondance des pluics a fait l'objet d'expériences prolongées à l'Ecole forcstière de Nancy $\left({ }^{2}\right)$, et toutes les déterminations faites en France comme à l'étranger montrent que la quantité d'eau pluviale est augmentée de plus d'un dixième par l'état boisé du sol.

Condensations occultes. - Le reboiscment accroît en outre dans une large mesure les condensations occultes, rosée, brouillard, geléc blanche, auxquelles le pluviomètre est insensible, et dont l'apport n'avait pas été mis en ligne de comple quand le professeur Fonec montra qu'il est comparable à celui des pluies $\left({ }^{8}\right)$. Il explique par cet apport commenl le bassin du lac Léman débite un volume d'eau supéricur à celui qu'y déversent les pluies et los neiges ; et cel excédent d'écoulement n'est pas un fait isolé, car il a été constaté dans d'autres bassins par les météorologistes suisses $\left(^{4}\right)$ et dans un bassin du plateau de Langres, qui alimente lo réservoir de la Mouche, par l'ingénieur en chef Morssenet. Pour qu'un bassin débite plus d'eau 'qu'il n'en reçoit des phuies mesurées au pluviomètre, il faut iqu'il reçoive d'une autre origine une quantité d'eau suffisante pour compenser les pertes que subisscint les caux pluviales par suite de l'éraporation el de l'absorption dans los partics fissurées du sol. L'évaporation faisant à elle seule disparaitre plus que la moitié des eaux pluviales (5), l'apport supplémentaire étranger anx pluies ou neiges figure pour plus de moilié dans les caux débitées par le bassin et mesurées à sa sortie ; comme il est soumis aux mêmes causes d'évaporation ou d'absorption, on doit présumer que la quantité d'eau supplćmentaire reçue par ce bassin est supérieure à celle des caux pluvialcs. On ne connaît, d'ailleurs, d'autre origine à ces caux supplémentaires que les condensations occultes, dont le dépòt sc produit partout, et les résurgences qui pourraient exceptionnellement amener dans un bassin des eaux déversées par les pluies dans un autre ; de sorte que l'apport des condensations occultes est prédominant et parfois supéricur à celui des pluies.

Si l'on ne connaîl pas encore par des mesures précises la

(1) E.-A. Martel. La marche à la lune. Premier Congrès de l'arbre et de l'eau, page 355. Limoges 1907.

(2) DE DRouIN DE Bouville Observations de météorologie forestière a la station de recherches de l'Ecole nationale des eavix el foréts

bulletın du ministère de l'agriculture 1909, no 2.

(3) BOREL Le Lëman, monographie limnologique, Lausanne, $1892-1895$.

(i) Service d'études des grandes forces hydrauliques, région des Alpes. Paris 1908, III, page 22.

(5) Daubré. Commission des Inondations Rapports et documents, page 506. Paris'1910. Imprimerie Nationale. proportion dans laquelle le reboisement augmente ces condensations occultes, 11 suffit de remarquer les panaches de givre qui garnisscnt un arbre après certaines nuits d'hiver, et l'infime quantité de rosée qui recouve dans son voisinage le sol dénudé sur une surlace équiralente à sa projection horizontale, pour voir que la végćtation' augmente considérablement les condensations occultes.

Diverses Sociétés et Publications scientiliques se sont attachées depuis quẹlques années, des deux còtés de l'Atlanlique (l) à stimuler et à coordonner les recherches daus cet ordre d'idées. Les expériences comparalives que M. Marchañ avait projetées à l'observatoire du Pic du Midi ont été malheureusement ajournées par le décès de cet éminent météorologiste ; mais on sait déjà, par les obscrvations du capitaine Henri Polıer, que dans des régions sćnégalaises, ćloignées de la mer et des cour's d'eau permanents, la rosée subsiste pendant la saison sèche, assez abondante pour entretenir la végétation et abreuver certains animaux; par celles de sir William Garderer ReEd qu'en Californie où il ne pleut pas depuis le commencement de juin jusqu'à la fin de septembre, les arbres ruissellent d'humidité et les prairies restent vertes dans leur voisinage, au lieu de roussir comme en terrain découvert. Ces observations confirment suflisamment les indications de la théorie pour donner l'assurance qu'en reboisant les bassins supérueurs des torrents on $y$ doublerait au moins les condensations occultes. Les caux que reçoit chaque bassin seraient augmentées ainsi de moitić (50\%) par le renforcement des condensations occultes, en sus des ro \% dus à l'accroissement de la pluviosité sur les terrains boisés, et l'augmentation tolale serail ainsi de $60 \%$. On pourrait donc, tout en ne reboisant que le tiers de chaque bassin afin de laisser aux habitants des pâturages pour leurs troupeaux, relever de $20 \%$ le débit utilisable pour la produc lion des forces motrices. Sans doute ce résultat ne peut etre instantané ; mais, si le tiers des montagnes élait reboisé depuis quelques dizaines d'annérs, il aurait été possible d'utiliser un bien plus grand nombre de chules, et colles aménagces jusqu'à ce jour pour la houille blanche produiraient le travail de $x .800 .000$ chevaux-vapeur au lien de ז.500.000 seulement.

Il est bien regrettable que l'œuvre du reboisemenl, commencée par l'Etat depuis 1860 , ait été si longlemps entravée par l'opposition des montagnards, aveuglés par un déplorable préjugé d'antagonisme entre la forêt et le pâturage. Ce préjugé est maintenant déraciné, depuis que les expériences de "l'Association centrale pour l'aménagement des montagnes " $\left({ }^{2}\right)$ ont montré combien sont solidaires ces deux éléments essentiels de l'économie alpestre, depuis que ces

(1) P. 1)escombes. - Sur un mode de l'aclion hydrologique des forêts. Procès-verbaux de Ia Socıété des sciences physiques et naturelles de Bordeaux, 19 mars 1904. La défense forestiere el pastorale Paris 1911. Gauthier-Villars; éd. L'ulilité inlernalionale des forếls. La vie internationale, t. v. p. 27-57, Bruxelles 1914. - Les pluies, les condensalions occultes et le rebousement ralionnel - Mémoires de la Soclété des seiences physiques et naturelles, $t$ v. 60 . série Bordeaux 1914. - Le reboisement et les condensations occulles. Comptes rendus de l'Assocration francaise pour l'avancement des sciences, Congrc̀s du Havre 1911. - Entreprises utiles à l'amélioration du régime des eaux, 2c congrès de la houille blanche. Tome I, page 317 Lyon 1914. - Retor. slation andoccult condensalion Monthly Weather Review(43.617) Washington, 1915. - William Gardner Reed Fog as Source of waler supply Monthly Weater Review $(11,288)$ - D Varigny. Cinronique scientifique, la biblothòque untverselle, I ausanne 1917. - Influence du reboisement sur l'abondance des condensalions occultes. - Annales de la Socicté d'agriculture de la Gironde 1917, page 271. - El viejo Forestal, Conacn. sacion del algua delos nieblas, Bulletin de los amigos del arbol, mars 1918.

(2) Association désintéressée de souscripteurs a 10 francs par an, reconnue d'utilité publique, dont le siège est à Bordeaux, 142, rue de Pessac, 
lecons de choses font jaillir la vég'étation spontanée dans les plaies béantes du sol jusque sur les rochers (1) et transforment autour d'elles les montagnards en auxiliaires du reboisement.

la France est maintenant contrainte par l'agression préméditée de la barbarie à sacrifier ses forcts (2) pour coffrer ses tranchées sans enlever au transport des troupes et des munitions la flotte commcrciale qui lui apportait en temps de paix plus que moilié du bois d'œuvre nécessaire à ses industries ( ${ }^{3}$ ). Il lui faudra donc développer aussitót après la victoire finale un immense effort de restauration forestière, dont les éléments doivent être complétés dès aujourd'hui, pour ne pas tarir les sourees de la houille blanche et de toutes les branches de la richesse publique $\left.{ }^{4}\right)$.

\section{II. - LES EXEMPTIONS D'IMPOTS APRES REBOISEMENT (").}

On snit que la législation et la jurisprudence française sont bien loin d'être adaptées aux particularités de la propriété forestic̀re, dont les produits périodiques à longue échéance ne sauraicñt être confondùs avec les produits annuels de l'agriculture ni avec ceux, intermiltents, de la viticulture. Cetle différence fondámentale dans la nature des revenus apporte à l'assictte de l'impôt des difficultés déconcertantes, dont l'élude tient une large part dans le cours de sylvonomie (ćconomie et politique forcstières) fondé en rgra par l'Association centrale pour l'Aménagement des montagnes à la Faculté des sciences de Bordeaux.

Si la législation fiscale des forêts n'a pas encore-ćté modifiéc comme leur législation économique, où la loi du 2 juillel Igr 3 " tendant à favoriser le reboisement et la conservation des forêts privées " a posé la première assise pour l'orientalion des capitaux vers le reboisement, la jurisprudence fiscale des forêts vient do faire un pas en avant qui présenle un intérêt capital pour les propriétaires forcstiers de la Gironde.

Un Arrêté. - Le Conseil de préfecture des Landes vient en effet de prendre, le is décembre $19 \mathrm{I}_{7}$, l'arrêté suivant :

"Considérant qu'aux termes des lois du 3 r décembre I907 ct 29 mars 1014 , article 3 , le nonvel impót foncier des proprićtés non bâties est basé sur le revenu actuel des parcelles composant ices propriẹtés ; que, pour donner une base légale à la nouvelle évaluation forestière, il faut rechercher et déterminer le revenu des parcelles, soit au moment de lá nouvelle confection du cadastre, soit au moment de l'entrée en application de la loí du 29 mars r 9 rá, c'est-à-dire au plus tard le $3 \mathrm{x}$ décembre $\mathrm{r} 9 \mathrm{x} / \mathrm{4}$;

"Considérant que c'est méconnaître l'esprit de la loi et en fausser le sens ct la portće que d'attribuer à des parcelles boisées, manifestement improductives à raison de leur jeune âge, non le revenu net qu'elles peuvent actuellement produjire, mais le revenu net moyen qu'elles seraient susceptibles de rapporter pendant une période de soixante ans et de calculcr ainsi sur ce chiffe le quantum de l'impôl-actuelle-

(') Le reboisement et le développement économique de la France, Paris 1918. Berger-Levrault, éniteur.

(2) H. Gomot, Adieu, nos belles foréts! (Le " Petut Journal ", 9 janvier 1918.)

(3) La répercussion foresticire de la guerre. ("Bois et Résineux ", 26 mâi 1918)

(4) Le reboisement et le developpement economique de la France, Paris 1918, Berger-Levrault, éditeur. - Le deficit de la populalion dans les régions montagneuses. (Journal de la Sociéte de statistique de Paris, février 1917 )

(5) Extrait de Bois el Résineux, du 5 mai 1918. ment réclamé.; que c'est à bon droit que le pétitionnaire réclame un dégrèvement pour les parcelles boisées actucllement improductives de revenus. Après en avoir délibéré, Arrêle :

"Les parcelles improductives de revenus énumérés dans " la réclanation de $X \ldots$, seront dégrevécs de l'impôt auquel "elles ont élć assujelties pour l'annóe I917. "

Voila une décision que le Syndicat agricole des Landes n'a obtenue qu'après de longs efforts et des débats encore plus longs. Les sylvicultcurs girondins peuvent en faice leur profil.

Elle a ćlé résumée en deux lignes par un conseiller génćral de l'Aveyron, qui est en même temps conseiller à la Cour de cassation :

"Les bois de pins qui, après un premicr abatage, " sont replantés à main d'homme peuvent à nouveau " bénéficier de l'exemption trentenaire de l'article 226 dı " Code foresticr.

"Dans les bois de haute futaie qui ne sont pas mis en " coupes réglées, l'abatage futur" n'est pas un revenu acluol " pouvant servir de base à la contribution foncière (1).

Un Commentaire. - Il convient d'ajouter à cette formule si lucide quelques cxtraits du savant commentairc donné par son auteur :

"Les questions fiscales font, en ce moment, l'objet de toutes les préoccupations. L'aúgmentation dics impôls va aller en croissant et le poids de la gucre sera supporté par plusicurs génćrations de contribuables. Ce sera pour les gens de l'arrière l'accomplissement d'un devoir patriotique auquel nul ne doit se soustraire ; il ne faut pas se lasser de le répéter dans notre Rouergue où la morale fiscale est souvent fort relâcbée. Frauder le fisc est une pratique à lacruelle Jes casuistes les plus rigoristes ne trouvent rien à redire. Et cependant ne voit-on pas que se soustraire à sa quote-part légitime d'impôt, c'est la faire retomber sur le voisin ? Et faire payer à colui-ci ce qu'il ne doit pas, nest-ce pas manquer à la probité la plus élémentaire? Nous avons beaucoup à faire pour réformer notre mentalité aveyronnaise sur ce point. Mais ce n'est pas un sermon sur la probité fiscale que je veux écrire ici aujour'dhui.

"Je veux, au contraire, sur un point spécial et tout à fait nouveau, signaler à certains contribuables fort intẻressants jusqu'où va leur droit à l'exemption fiscale. Payer tout ce que l'on doit, au delà résister, telle devrait être la règle en celte délicatc matière.

"Dépuis quelques annéès, on a beaucoup planté ct replanté sur notre sol rouergat. Une Sociélé de reforestation, dont j'ai parlé ici-même, se fonde à Rodez et groupe fort utilement des bonnes volontés un peu éparses. Les terrains ainsi replantés sont-ils toujours, partout et à perpétuilé exempts de la contribution foncière? Tclle est la question qui vient d'être récemment et très solennellement débattue devant un Conseil de préfecture et dont je-voudrais dire un mot jici. Elle est d'autant plus intéressante que la loi d.u 3 I juillet Igr 7 a porté de 4 à $5 \%$ le taux de la contribution foncière des propipiétés bâties ou non bâties.

" J'article 226 du Code forestier porte que "les scmis et " plantations de bois sur le sommet et le penchant des mon" tagnes, sur les dunes et dans les landes seront exempts de " tout impôt pendant trente ans."

"Mais sur ces terrains ainsi plantés, l'Administration des Contributions directes a, en cerlaines régions, émis la dolible prétention : $\mathbf{x}$. De ne faire jouer l'arlicle $2_{2} 6 \mathrm{du}$ Code

(1) Billet Rouergal, journal de l'A veyron, 3 mars 1918. 
forestier qu'une seule fois, de telle sonte qu'après trente ans écoulés el après compe, si le proprictare d'une foret est obligé de la replanter, il n'aura plus droit à aucune nouvelle excmption; 2. de réparlir le produit dos coupes sur les années qui les précedent. Exrmple : les pins sonl abaltus généralement à l'àge de 60 ans. On considécera pour la lixation du revenu foncier que la foret de pins donne chaque année, à partir de la plantalion, un $60^{\circ}$ de la valcur de la coupe.

"La premiere de ces prélentions est sans infrect pour les forêts de hêtres ou de chênes qui se reproduisent automaliquenenl elles-mones el sans aucun travail spécial. Là, il ne saurat etre question d'une nouvelle plantation et on conprend que l'arlicle $2: 6$ du Code forestier ne puisse jouer qu'une fois al pour une seule période de trente ans. Leur adoleseence, au point de vue fiscd, no dure que trente ans, mais leur maturitó reste élernelle el ne comnail aucune décrépitude.

"La première de, questions que je signalais loul à l'benre ne se pose done que pour les forèts de pins qui, après coupe, doivent ètre replaulées. Les jeunes pins ne croissent pas sous les grands arbres ; ceux que lon pourtait y semer ou y planter, privés d'aix ou de lumière, ne poutraient sc développer ; les troncs ni les racines né donnent aucun rejel, de lelle sorte que lorsque les pins qui forment me forêt ont dé coupés, il ne reste souvent rien et il fant la reconstituer de toutes pièces. Le proprićtaire est alor's obligé de renouveler l'efforl qu'il avall fait une première fois. Bénéficiera-t-il d'une scconde exemption trentenaire de la contribution foncière en vertu de l'article 226 du Gode forestier ? Non, disaif J'Administration des Contributions directes ; oui, vient de répondre le Conseil de préfecture des landes par son arrèté du 18 dóconibre 1917 ."

\section{GIRONDINS A L'GUVRE!}

Il appartient maintenant aux proprićtaires forestiers de la Gironde, dont.le geńre d'exploitation est identique à celui du département voisin, de manouvrer en vue d'attcindre les mèmes exonćrations.

Gette nourelle amélioration de' la jurisprudence fiscalé ne présente pas moins d'importance que celle obtcnue pour remédier pendanl le reste de la période trentenaire à l'ajournemenl si fréfuent des demandes nécessaires pour profiter du dégrèvement prévu par le Code forestier âprès un reboisement de landes $\left({ }^{1}\right)$.

\section{III. - UN VQEU DEE L'ASSOCIATION CENTRALE POUR L'AMÉNAGEMENT DES MONTAGNES (?)}

La France est aujourd'hui contrainte, par les nécessités de la défense nationals, à sacrifier ses forits pour coffrer ses lanchées sains enlever an transport des trompes et des munilions la flotte commorciale qui lui apportait, en temps de paix, plus de la moité du*bois d'ceuve indispensable à ses industries. 11 lui faudra donc développer aussitòt après la victoire finale un immense effort de restauration sylvestre, dont lous les éléments doivent ctro complétés dès mainlelianl, pour ne pas farir les sources de la houille blanche el toules les branches de la riohesse publique.

Dans ces conditions, l'Association centrate pour l'Áménigement des montanges, doml les expériences de démonstration sylvo-pastorale sont aulant de leçons de choses

(1) lixonémbion djmpôl sur les terrains spmés on plantés en hois Innoles de la Sociéli dingricullure de la Gironde, 1915, page 55.

(2) Bxtmit de Bois el liésinew\%, du 11 juillel 1918. remplaçant la dégradation par la régénćration spontance d transformant les montagnards en auxiliares du reboisement, dont les publicalions et le contrs de sylvonomie à la Faculté de Bordeaux analysent les particularités déconcritantes de la proprićté sylvestre et développent leo bases de la politicue forestière adoptée par la loi du a juillet 1913 , issue de son initialive, a émis le voeu suivant :

\section{Délibérattoy du Í Jun I 98 .}

Le Comité de l'Association centrale pour l'Aménagement des montagnes : Considérant l'urgente nécessité de remplacer les arbres sacriliés à la Défense nationale ;

Considérant que les Sociétés forestières el les particuliers, propriétaires des deux tiers des forèts frangaises doivent être mis ch mesure de collaborer immédiatement à cetle ouve indizpensable ;

Considérant que la sylviculture privée col encore dúpourvue des encouragements ef du crédit organisés pour les autres branches de l'agriculture ou de l'industric ;

Considérant que da loi du 2 juillet rgr3, tendant à favoriser le reboisement et la conservation des forêts privées, a déjà débarrassé le reboiscment d'importants obstacles en autorisanl les Associations et les Caisses d'éparone à y prendre part, mais qu'il subsiste encore des dispositions législalives ou afministratives empêchant diver-es calégories de capitaux d'y participer dans la mesure nécessaire. Emet le voeu :

"Que les pouvoirs publics dolent sans retard la sylviculture privée d'encouragements et d'instruments de crédit équivalents à ceux existants déià pour les aulres branches de l'agricullure ou de l'industrio, et fassent disparaitre les obstacles législatifs ou administratifs qui gênent encore le reboisement. ")

Fit décide que la présente délibération sera transmise à M. le ministre de la guerre, président du Conseil et à MM. les ministres de l'agriculture, des Iravaux publics, des finances, de l'armement, du blocus, de l'intérienr, du commerce, du travail ef de la prévoyance socialé.

Paul Descombes, Directeur honoraire des Manufactures de lElat

\section{COIIMISSION EXTRAPARLEMENTAIRE DES FORGES HYDRAULIQUES}

\section{Textes des Procès-Verbaux des Séances}

\section{DEUXIĖME SÉANCE DU $21^{\circ}$ JUIN $1917^{\text {(14 }}$ SÉANCE)}

Présents : MN. KLot\%, Président ; Drveldx, Léon Perrina, Margaine, Dutiors, Bertuélemy, Rousseau, Féret du Longbors, Mamiev, Salles, Dibut; Troté, Blazeix, Cinhmeil, Dupoxteil, Colonel Marson, Commandant Ciner, Inrrer, Conte, Conder, Bouchayer, Bovguult, Pivot, Lavand, Perit, Lomtre.

Excusés: MN. Monestren, Barte, Jecr, Alexandre Bérand, Marc RéviLte.

La séance est ouverte à 9 heures du soir.

M. Le Président dit que, sur la première question à résoudre ee soir par la Commisson : "Quelle participalion dans les redevances faul-il assurer aux collectivités? ", M. Mahicu a préparé une nole dont il donme leclure :

"A l'heure achuelle, les colleclivilés diverses n'ont droit a aucune part des redeyances on argent qui ront entiorement à l'Etat. Par contre, elles brnéficient d'un aceroissement de revenus sous forme d'impôts directs el en particulier de la patente, an 